\title{
Lessons learned from administration of high-dose methylprednisolone sodium succinate for acute pediatric spinal cord injuries
}

\author{
Michelle C. Caruso, PharmD, BCPS, ${ }^{1}$ Margot C. Daugherty, MSN, MEd, RN, CEN, ${ }^{2}$ \\ Suzanne M. Moody, MPA, ${ }^{2}$ Richard A. Falcone Jr., MD, MPH, ${ }^{2}$ Karin S. Bierbrauer, MD, ${ }^{3}$ and \\ Gary L. Geis, MD4
}

Divisions of ${ }^{1}$ Pharmacy, ${ }^{2}$ Trauma Services, ${ }^{3}$ Neurosurgery, and ${ }^{4}$ Emergency Medicine and Center for Simulation and Research, Cincinnati Children's Hospital Medical Center, Cincinnati, Ohio

\begin{abstract}
OBJECTIVE Methylprednisolone sodium succinate (MPSS) has been studied as a pharmacological adjunct that may be given to patients with acute spinal cord injury (ASCI) to improve neurological recovery. MPSS treatment became the standard of care in adults despite a lack of evidence supporting clinical benefit. More recently, new guidelines from neurological surgeon groups recommended no longer using MPSS for ASCl, due to questionable clinical benefit and known complications. However, little information exists in the pediatric population regarding MPSS use in the setting of ASCl. The aim of this paper was to describe steroid use and side effects in patients with ASCl at the authors' Level 1 pediatric trauma center in order to inform other hospitals that may still use this therapy.
\end{abstract}

METHODS A retrospective chart review was conducted to determine adherence in ordering and delivery according to the guideline of the authors' institution and to determine types and frequency of complications. Inclusion criteria included age $<17$ years, blunt trauma, physician concern for $\mathrm{ASCl}$, and admission for $\geq 24$ hours or treatment with high-dose intravenous MPSS. Exclusion criteria included penetrating trauma, no documentation of ASCl, and incomplete medical records. Charts were reviewed for a predetermined list of complications.

RESULTS A total of 602 patient charts were reviewed; 354 patients were included in the study. MPSS was administered in 59 cases. In 34 (57.5\%) the order was placed correctly. In $13(38.2 \%)$ of these 34 cases, MPSS was administered according to the recommended timeline protocol. Overall, only $13(22 \%)$ of 59 patients received the therapy according to protocol with regard to accurate ordering and administration.

Among the patients with $\mathrm{ASCl}, 20$ (55.6\%) of the 36 who received steroids had complications, which was a significantly higher rate than in those who did not receive steroids (8 [24.2\%] of 33, p = 0.008). Among the patients without ASCI, $10(43.5 \%)$ of the 23 who received steroids also experienced significantly more complications than patients who did not receive steroids (50 [19.1\%] of $262, p=0.006)$.

CONCLUSIONS High-dose MPSS for ASCI was not delivered to pediatric patients according to protocol with a high degree of reliability. Patients receiving steroids for pediatric ASCI were significantly more likely to experience complications than patients not receiving steroids. The findings presented, including complications of steroid use, support removal of high-dose MPSS as a treatment option for pediatric ASCl.

https://thejns.org/doi/abs/10.3171/2017.7.PEDS1756

KEY WORDS spinal cord injury; pediatric; methylprednisolone; trauma; neck injury; spine

$\mathrm{T}$ REATMENT options for acute spinal cord injury (ASCI) are limited. Methylprednisolone sodium succinate (MPSS) has been studied as a pharmacological adjunct that may be given postinjury to improve neurological recovery. MPSS possesses a variety of neuroprotective effects, including inhibition of lipid peroxidation, calcium influx, and ischemia and antiinflammatory activity. ${ }^{6}$ The National Acute Spinal Cord Injury Study (NASCIS) trials, beginning in the mid-1980s, reported improved motor and sensory scores at follow-up after treatment with high-

ABBREVIATIONS AANS = American Association of Neurological Surgeons; $\mathrm{ASCl}=$ acute spinal cord injury; CNS = Congress of Neurological Surgeons; EMR = electronic medical record; GI = gastrointestinal; ICU = intensive care unit; ISS = Injury Severity Score; MPSS = methylprednisolone sodium succinate; NASCIS = National Acute Spinal Cord Injury Study; $\mathrm{SCl}=$ spinal cord injury.

SUBMITTED January 30, 2017. ACCEPTED July 6, 2017

INCLUDE WHEN CITING Published online October 6, 2017; DOI: 10.3171/2017.7.PEDS1756. 
dose MPSS. After NASCIS II and NASCIS III, high-dose MPSS therapy became widely considered as a standard of care for ASCI.

Criticisms of the NASCIS trials included study designs, data quality, statistical analysis, interpretation, and conclusions. .,4, $, 10,11$ The primary outcome analyses of all 3 NASCIS trials have shown no clinical benefit, and the only positive effects seen with high-dose MPSS therapy were determined through post hoc subgroup analysis. Subsequent studies have raised concerns regarding the safety of this therapy in light of the results from the NASCIS trials and the known complications of high-dose steroids. ${ }^{2,5,7,9,12}$

In 2002, due to insufficient evidence, the American Association of Neurological Surgeons (AANS)/Congress of Neurological Surgeons (CNS) Joint Section of Disorders of the Spine and Peripheral Nerves recommended the use of MPSS as an option, not as a guideline or standard. ${ }^{1}$ Given the lack of data regarding the use and complications of MPSS among pediatric patients, a multidisciplinary consensus guideline for use at our institution was implemented for the administration of this therapy in ASCI when the following criteria were met: 1) blunt mechanism of injury, 2) patient presentation within 8 hours of injury, and 3) presence of neurological and/or sensory deficit(s) (symptoms of paresthesia alone did not qualify). If the patient was transferred from an outside facility and MPSS administration had already been initiated, this would be completed per guidelines, even in the presence of a negative MRI study.

Therapy at our institution followed the NASCIS II and NASCIS III protocols for dosing and administration, which consisted of methylprednisolone administration with a bolus dose followed by a 45-minute pause and then a 23-hour continuous infusion. The dose and administration instructions remained the same during the time of the study. The unusual administration requirements for this therapy were found to present challenges with prescribing, given the various order entry systems in place at our institution, dispensing by the pharmacy, and administration and monitoring by nursing personnel.

To the best of our knowledge, no studies currently exist describing problems associated with prescribing or administering high-dose MPSS therapy. To address this gap in the literature, we studied the medication use process for this treatment at our institution. Our specific aims were to determine 1) adherence in ordering and delivery of medications according to the guideline and 2) the types and frequency of complications.

\section{Methods \\ Study Design}

This was a retrospective, cross-sectional cohort chart review of pediatric trauma cases involving patients who presented to our institution from January 1, 2000, through September 30, 2010. The Cincinnati Children's Hospital Medical Center institutional review board approved the protocol for the study prior to commencement.

\section{Setting \\ Our institution is a 643-bed academic tertiary-care}

facility located in southwestern Ohio. The trauma center is an American College of Surgeons-designated Level 1 pediatric center and admits, on average, 2100 trauma patients annually.

\section{Patient Population}

The institutional Trauma Registry was queried for patients who met the standard inclusion criteria for ICD-9 injury codes 800-959. We further limited the ICD-9 codes to 806,847 , and 952-953, which were indicative of possible ASCI. Patients were included if they were less than 17 years of age, presented with blunt trauma, had a documented physician concern for ASCI, and were either admitted for $\geq 24$ hours or received MPSS for ASCI (initiated at our institution or a referring hospital). Exclusion criteria included penetrating trauma, no documentation of ASCI, and incomplete medical records.

\section{Patient Data}

Patient information was retrospectively collected from the Trauma Registry and included demographics (age, sex, and race), length of stay, mechanism of injury, date/time of injury, referring hospital information, Injury Severity Score (ISS), discharge diagnosis, outcome (alive vs deceased), and discharge disposition.

Electronic medical record (EMR) reports, including EmSTAT (Version 1.30), WORx (Version 3.4), and EPIC (Version Spring 2008), were run to determine if any of the patients from the Trauma Registry query had orders for MPSS during their visit. Paper charts were requested for patients treated prior to implementation of the EMR system. Charts were manually reviewed by the trauma nurse educator and emergency medicine clinical pharmacist for the following data elements: steroid treatment, including guideline adherence; gastrointestinal (GI) ulcer prophylaxis; and a predetermined list of side effects attributed to steroid use. Data were entered into a database, and interrater reliability was assessed on $20 \%$ of the charts to ensure accuracy.

\section{Statistical Analysis}

Descriptive frequencies were generated for patient demographics, guideline adherence, and complications. Categorical and binary variables were summarized by frequency (\%), and continuous variables were summarized by median and range. Statistical comparisons between cohorts (i.e., those who received steroids and those who did not) were analyzed using the chi-square test with $\mathrm{p}<0.05$ considered significant.

\section{Results}

During the 10-year study period, 20,223 injured patients were treated at our institution. Applying our inclusion criteria and the aforementioned ICD-9 codes yielded 602 patient records. A total of 248 charts were excluded for the following reasons: age (17 cases), incomplete medical records (32 cases), length of stay $<24$ hours without administration of MPSS (187 cases), no ASCI suspected (7 cases), or penetrating trauma (5 cases). Of the 354 in- 


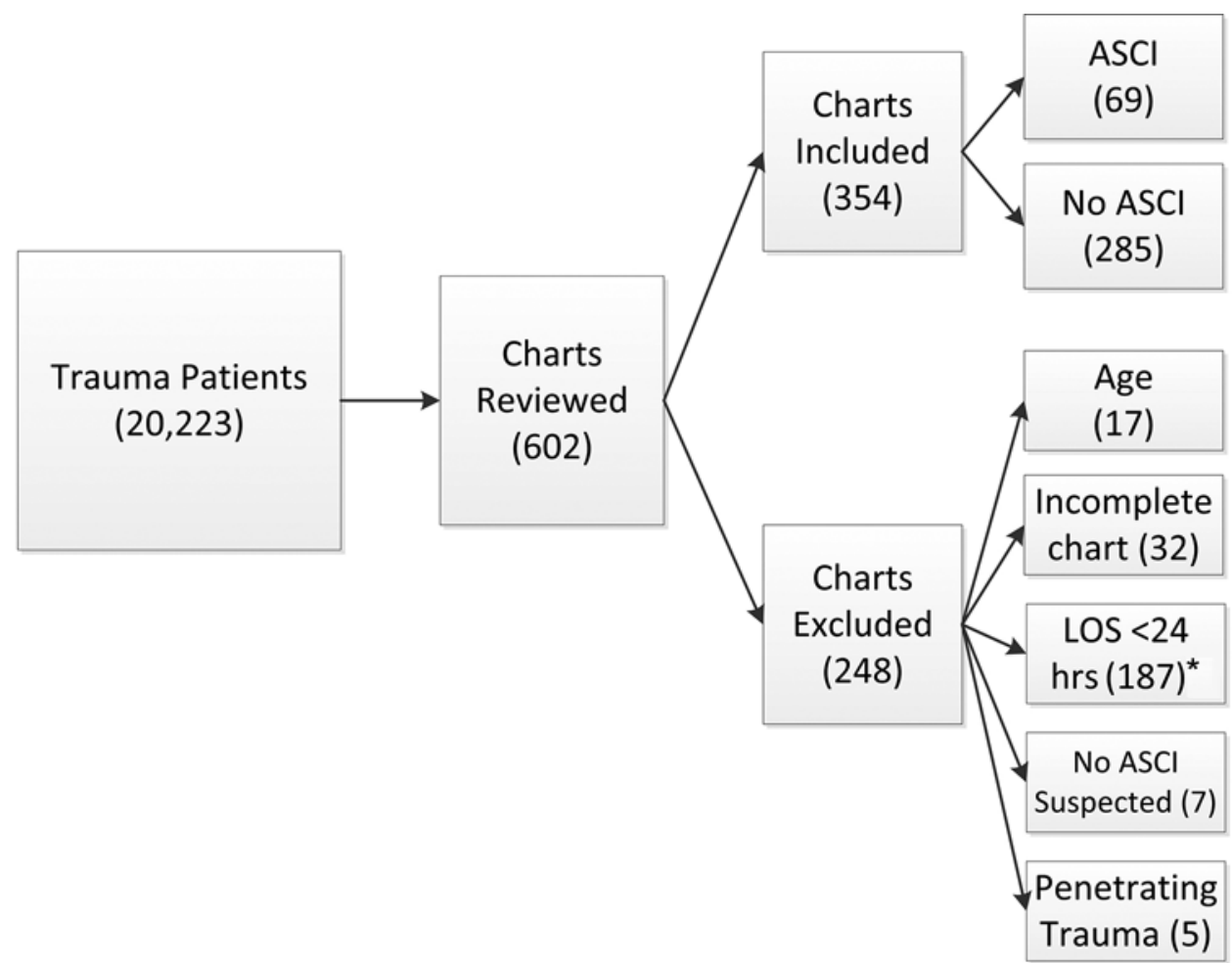

FIG. 1. Inclusion and exclusion summary. Values in parentheses indicate numbers of patients/charts. LOS $=$ length of stay. ${ }^{*} \mathrm{~Pa}$ tients with LOS $<24$ hours were excluded only if they did not receive MPSS.

cluded charts for patients 0-17 years of age, 69 (19.5\%) showed an eventual diagnosis of ASCI (ICD-9 codes of $806,952$, or 953$)$, while the remaining $285(80.5 \%)$ did not (Fig. 1). Of the patients with ASCI, $36(52.2 \%)$ received steroids. Of those without ASCI, $23(8.1 \%)$ received steroids. This resulted in a total of 59 patients $(16.7 \%)$ treated with high-dose MPSS (Fig. 2).

The average age of all patients included in this study was 11.9 years (range $0-17$ years). The average length of stay was 5.3 days (range 1-51 days). More of the patients were male than female, and the majority were white. No significant differences in demographics were noted between patients receiving steroids and those not receiving steroids. No significant differences were noted in demographics between patients with and without ASCI. Patients who received steroids, both those with and without ASCI, had lower average Injury Severity Scores (ISSs) and length of hospital stay than those not receiving steroids (Table 1).

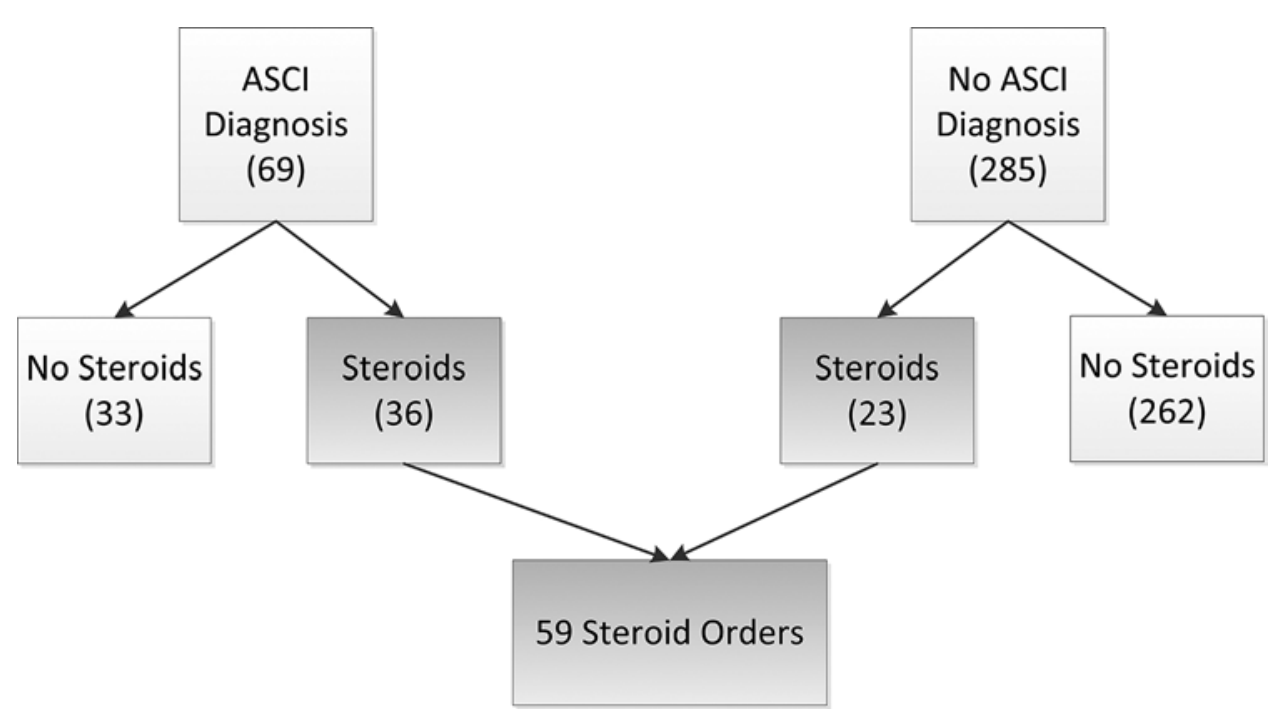

FIG. 2. ASCI status and steroid exposure with resulting steroid orders. 


\section{Medication Ordering and Administration}

Given the complicated nature of ordering and administration of this therapy, the accuracy of the ordering process was investigated. The 3 components of the medication order investigated included drug, dose, and route. As a composite outcome, the therapy was ordered correctly for the purposes of this study in $57.6 \%$ of cases $(n=34)$. The medication was ordered correctly as MPSS for 53 (89.8\%) patients. Four $(6.8 \%)$ patients had additional steroids ordered after the completion of MPSS therapy, and in 2 cases $(3.4 \%)$ the drug was unable to be determined. The correct dose was ordered per guideline for 41 (69.5\%) patients; there were 12 orders (20.3\%) with incorrect dosing, and for 6 orders (10.2\%) the dosing could not be determined. The route of administration was correctly ordered as intravenous for $58(98.3 \%)$ orders and could not be determined due to lack of documentation in the medical record for one order (1.7\%) (Table 1).

Of the 59 cases in which patients received MPSS, the order was placed correctly in $34(57.6 \%)$. In 13 (38.2\%) of these 34 cases, the medication was administered following the recommended timeline protocol, while in $15(44.1 \%)$ it was not administered per the guideline. Due to lack of medication administration record (MAR) data, 6 (17.6\%) of the orders could not be assessed for administration details.

Overall, only $13(22 \%)$ of 59 patients received the therapy according to protocol with regard to accurate medication ordering and administration (Table 1).

Of the patients ordered MPSS, 38 (64.4\%) were also ordered GI ulcer prophylaxis with an $\mathrm{H} 2$ blocker or proton pump inhibitor per guideline; 21 (35.6\%) of the patients did not receive an order for this therapy (Table 1).

\section{Patient Complications}

Among the patients with ASCI, 20 (55.6\%) of the 36 who received steroids had complications, which was a significantly higher rate than in the patients with ASCI who did not receive steroids $(8$ [24.2\%] of $33, p=0.008)$. In the group without ASCI, 10 (43.5\%) of the 23 who received steroids experienced complications, which again was a significantly higher rate than the corresponding rate in the patients without ASCI who did not receive steroids (50 [19.1\%] of 262, $\mathrm{p}=0.006$ ) (Table 1).

The frequency of complications in the pediatric patients who received steroids is shown in Table 2. Table 3 shows the frequency of complications among patients with and without ASCI and with and without steroid exposure. Patients receiving steroids experienced the highest numbers of complications per individual, as many as 6 complications in a patient with ASCI and 9 complications in a patient without ASCI.

Table 4 shows the types and frequency of specific complications in patients with and without ASCI and with and without steroid exposure. On average, pediatric patients receiving steroids had 2.3 complications per patient compared with 1.8 complications per patient in those not receiving steroids. In patients receiving steroids, the most common complications were nausea/vomiting, hyperglycemia, bradycardia, fever, and hypertension. In patients not receiving steroids, the most common complications were nausea/vomiting, fever, hypertension, an increase in white blood cell count, and bradycardia.

\section{Discussion}

ASCI is a critical injury in the pediatric and adult populations, with the potential for long-term disability for the patient and significant costs for the family and health care community. It is only natural that health care providers would explore and implement therapies at the bedside early in the management of suspected ASCI with the goal of reducing long-term morbidity. In 2002, the AANS/CNS Joint Section on Disorders of the Spine and Peripheral Nerves recommended the use of MPSS as an option, not as a guideline or standard. ${ }^{1}$ Our institution recognized this recommendation, realizing it was not targeted toward pediatric patients. After thorough evaluation, the multidisciplinary consensus was to incorporate clinical concern and patient status as factors in decision making for administration of MPSS for ASCI for pediatric patients presenting to our institution, following the institutional guideline. However, the complex nature of the high-dose MPSS protocol, with multiple inclusion criteria and a complicated dosing regimen, contributes to the unlikeliness that the health care team will implement the protocol exactly as it was intended, especially when it is applied infrequently.

Thus, it comes as no surprise that in our study of those patients at a Level 1 trauma center with a clinical suspicion of ASCI and MPSS ordered, only 13 (22\%) of 59 had correct order and administration of steroids per the guideline. Of the 69 patients ultimately diagnosed with ASCI, only $8(11.6 \%)$ received the correct order and administration of steroids per the guideline. While we were not surprised to find that the adherence to the guideline was under $100 \%$, we were surprised at how few times the therapy was administered as intended, especially given the intense institutional focus on patient and medication safety with pediatric-specific resources in place, such as the EMR system. Similar problems with inaccurate administration of this protocol in adults have been shown. In a study by Lee et al., only $42 \%$ of patients (58 of 138) were given MPSS correctly according to the NASCIS II and III protocols. ${ }^{6}$ Other studies, which describe complications associated with high-dose MPSS for ASCI, do not discuss ordering or administration errors. ${ }^{712}$ In our study, the results for accurate ordering and administration were lower than reported by Lee et al., which could be due to the population (pediatric) served and the low frequency with which this protocol was used in our population. Based on the findings of poor compliance with the protocol, a worksheet was created to assist staff with timing of administration of the bolus and continuous infusion of MPSS. GI prophylaxis was also incorporated into the worksheet as a reminder to order in the EMR. An electronic health record order-set was also created (once the EMR system was implemented), which automated the dose and timing in the EMR.

Given the infrequent or uncommon nature of ASCI in our pediatric population, it makes sense for providers to look for algorithms, guidelines, or protocols to simplify and prescribe care. However, there is innate danger when applying these protocols if a patient does not meet inclu- 
TABLE 1. Demographics, steroid usage, and guideline compliance

\begin{tabular}{|c|c|c|c|c|c|}
\hline \multirow[b]{2}{*}{ Characteristic } & \multirow{2}{*}{$\begin{array}{l}\text { All Patients } \\
(n=345)\end{array}$} & \multicolumn{2}{|c|}{ ASCI ICD-9 Codes } & \multicolumn{2}{|c|}{ No ASCI ICD-9 Codes } \\
\hline & & w/ Steroids $(n=36)$ & No Steroids $(n=33)$ & $w /$ Steroids $(n=23)$ & No Steroids $(n=262)$ \\
\hline \multicolumn{6}{|l|}{ Sex } \\
\hline Male & $230(65.0)$ & $26(72.2)$ & $24(72.7)$ & $15(65.2)$ & $165(63.0)$ \\
\hline Female & $124(35.0)$ & $10(27.8)$ & $9(27.3)$ & $8(34.8)$ & $97(37.0)$ \\
\hline \multicolumn{6}{|l|}{ Race } \\
\hline White & $267(75.4)$ & $27(75.0)$ & $20(60.6)$ & $20(87.0)$ & $200(76.3)$ \\
\hline Black & $59(16.7)$ & $7(19.4)$ & $9(27.3)$ & $2(8.7)$ & $41(15.6)$ \\
\hline Multiracial & $5(1.4)$ & $0(0.0)$ & $1(3.0)$ & $0(0.0)$ & $4(1.5)$ \\
\hline Other & $21(5.9)$ & $2(5.6)$ & $3(9.1)$ & $1(4.3)$ & $15(5.7)$ \\
\hline Hispanic & $2(0.6)$ & $0(0.0)$ & $0(0.0)$ & $0(0.0)$ & $2(0.8)$ \\
\hline \multicolumn{6}{|l|}{ Age in yrs } \\
\hline Mean & 11.9 & 13.2 & 10 & 14.5 & 11.8 \\
\hline Range & $0-17$ & $5-17$ & $0-16$ & $9-17$ & $0-17$ \\
\hline \multicolumn{6}{|l|}{ LOS in days } \\
\hline Mean & 5.3 & 4.7 & 9.2 & 3.4 & 5 \\
\hline Range & $1-51$ & $1-27$ & $1-32$ & $1-16$ & $1-51$ \\
\hline \multicolumn{6}{|l|}{ ISS } \\
\hline Mean & 12 & 11 & 16 & 5.3 & 12 \\
\hline Range & $1-50$ & $1-34$ & $1-43$ & $1-50$ & $1-45$ \\
\hline Steroids for SCl & $59(16.7)$ & $36(100.0)$ & $0(0.0)$ & $23(100.0)$ & 0 \\
\hline \multicolumn{6}{|l|}{ Ordered per guideline } \\
\hline Yes & $34(57.6)$ & $22(61.1)$ & NA & $12(52.2)$ & NA \\
\hline No & $18(30.5)$ & $11(30.6)$ & NA & $7(30.4)$ & NA \\
\hline Not sure & 7 (11.9) & $3(8.3)$ & NA & $4(17.4)$ & NA \\
\hline \multicolumn{6}{|l|}{ Med per guideline } \\
\hline Yes & $53(89.8)$ & $33(91.7)$ & NA & $20(87.0)$ & NA \\
\hline No & $4(6.8)$ & $2(5.6)$ & NA & $2(8.7)$ & NA \\
\hline Not sure & $2(3.4)$ & $1(2.8)$ & NA & $1(4.3)$ & NA \\
\hline \multicolumn{6}{|l|}{ Dose per guideline } \\
\hline Yes & $41(69.5)$ & $26(72.2)$ & NA & $15(65.2)$ & NA \\
\hline No & $12(20.3)$ & $6(16.7)$ & NA & $6(26.1)$ & NA \\
\hline Not sure & $6(10.2)$ & $4(11.1)$ & NA & $2(8.7)$ & NA \\
\hline \multicolumn{6}{|l|}{ Route per guideline } \\
\hline Yes & $58(98.3)$ & $35(97.2)$ & NA & $23(100.0)$ & NA \\
\hline No & $0(0.0)$ & $0(0.0)$ & NA & $0(0.0)$ & NA \\
\hline Not sure & $1(1.7)$ & $1(2.8)$ & NA & $0(0.0)$ & NA \\
\hline \multicolumn{6}{|c|}{ Administered per guideline } \\
\hline Yes & $13(38.2)$ & $8(36.4)$ & NA & $5(41.7)$ & NA \\
\hline No & $15(44.1)$ & $10(45.5)$ & NA & $5(41.7)$ & NA \\
\hline Not sure & $6(17.6)$ & $4(18.2)$ & NA & $2(16.7)$ & NA \\
\hline \multicolumn{6}{|l|}{ Ulcer med given } \\
\hline Yes & $38(64.4)$ & $25(69.4)$ & NA & $13(56.5)$ & NA \\
\hline No & $21(35.6)$ & $11(30.6)$ & NA & $10(43.5)$ & NA \\
\hline \multicolumn{6}{|l|}{ Complications } \\
\hline Yes & $88(24.9)$ & $20(55.6)$ & $8(24.2)$ & $10(43.5)$ & 50 (19.1) \\
\hline No & $266(75.1)$ & $16(44.4)$ & $25(75.8)$ & $13(56.5)$ & $212(80.9)$ \\
\hline
\end{tabular}

LOS = length of stay; med = medication; NA = not applicable.

Numbers in parentheses represent percentages. 
TABLE 2. Frequency of complications in the 59 patients who received steroids

\begin{tabular}{cc}
\hline No. of Complications & Frequency $^{*}$ \\
\hline 1 & 13 \\
\hline 2 & 8 \\
\hline 3 & 4 \\
\hline 4 & 2 \\
\hline 5 & 1 \\
\hline 6 & 1 \\
\hline 7 & 0 \\
\hline 8 & 0 \\
\hline 9 & 1 \\
\hline * Frequency (number of patients) for the specified number of complications
\end{tabular}

sion criteria. In this study, there were 23 patients who ultimately were determined to not have ASCI who received high-dose MPSS therapy, and 10 (43.5\%) of these patients had complications. Additionally, while protocols are useful to standardize care for patients with similar conditions, Chang et al. summarize reasons why protocols are less likely to be followed by clinicians when they are complex or difficult to follow: 1) clinicians resist them, 2) protocols are inadvertently simplified so they do not apply to a wide range of patient populations, and 3) they no longer account for complex physiology. ${ }^{3}$ In this study, where over the 10year study period high-dose MPSS was usually considered standard therapy, 33 (47.8\%) of 69 patients with ICD-9 codes for ASCI did not receive steroids, which supports the initial point of Chang et al. To their second point, in the current study, patients receiving MPSS had lower ISSs, on average, than those who did not receive MPSS. We believe that this is because those with higher ISSs likely had multisystem trauma or may have been unresponsive. In patients with lower ISSs, the primary injury most often was isolated to the cervical spine, with associated neurological symptoms, making the team more likely to focus on the primary injury and administer steroids. This is an important point to take into consideration when future treatments for ASCI are developed.

High-dose MPSS therapy is not without complications. The majority of studies describing complications associated with this therapy were conducted in adult patients. A study by Suberviola and colleagues in 2008 examined the early complications of MPSS in ASCI patients. ${ }^{12}$ This was a retrospective evaluation of ASCI patients admitted to an intensive care unit (ICU) between January 1994 and December 2005. Patients were grouped according to presence $(n=59)$ or absence $(n=23)$ of MPSS treatment. There were no significant differences at baseline with respect to sex ratio, age (range 20.7-64 years), or degree of neurological deficit at ICU admission between the 2 groups. The MPSS group had an increase in respiratory tract infections (OR 8.19,95\% CI 1.10-358.6) and total infections $(p=0.004)$. Hyperglycemia occurred in $52(88 \%)$ of the 59-MPSS treated patients compared with $7(30 \%)$ of the 23 patients who were not treated with MPSS (OR 17.0, 95\% CI 4.52-66.3). Additional potential adverse effects of high-dose MPSS treatment in trauma patients include an increased incidence of pulmonary embolism, GI
TABLE 3. Frequency of complications by $\mathrm{ASCl}$ and exposure to steroids

\begin{tabular}{ccccc}
\hline $\begin{array}{c}\text { No. of } \\
\text { Complications }\end{array}$ & $\begin{array}{c}\text { ASCI \& } \\
\text { Steroids } \\
(\mathrm{n}=20)\end{array}$ & $\begin{array}{c}\text { No ASCI \& } \\
\text { Steroids } \\
(\mathrm{n}=10)\end{array}$ & $\begin{array}{c}\text { ASCI \& } \\
\text { No Steroids } \\
(\mathrm{n}=8)\end{array}$ & $\begin{array}{c}\text { No ASCI \& } \\
\text { No Steroids } \\
(\mathrm{n}=50)\end{array}$ \\
\hline 1 & 10 & 3 & 3 & 30 \\
\hline 2 & 3 & 5 & 3 & 8 \\
\hline 3 & 3 & 1 & 1 & 6 \\
\hline 4 & 2 & 0 & 0 & 5 \\
\hline 5 & 1 & 0 & 1 & 1 \\
\hline 6 & 1 & 0 & 0 & 0 \\
\hline 7 & 0 & 0 & 0 & 0 \\
\hline 8 & 0 & 0 & 0 & 0 \\
\hline 9 & 0 & 1 & 0 & 0 \\
\hline
\end{tabular}

The $n$ value given under each column head is the total number of patients in that group (column) who experienced a complication.

hemorrhage, and pancreatitis; a worsening of head injury outcome; the possibility of a missed hollow viscus injury due to "masking" of abdominal signs; and myopathy. ${ }^{6,7,12}$ Our pediatric data, with much larger numbers, mirrors these adult data, as we had an overall complication rate of $24.9 \%$ in the 354 studied patients, with the steroid cohort (with and without ASCI) having a 50.8\% complication rate compared with a complication rate of $19.7 \%$ in the nonsteroid cohort (with and without ASCI) (Table 1) (p $<0.05$ ). Complications in our study included respiratory, wound, or urinary tract infections in 10 (17\%) of the 59 pediatric patients who received steroids and hyperglycemia in $11(18.6 \%)$ (Table 4). Additional complications associated with the use of MPSS in ASCI include longer hospital stays and higher acute care charges. ${ }^{7}$ These complications frequently require an intervention or additional reassessments and monitoring; therefore, we believe all complications were worth reporting. However, in our pediatric patients who received steroids, we saw a shorter length of hospital stay (Table 1). As discussed above regarding injury severity and ISS scores, this may be reflective of the multisystem injuries sustained causing both lower recognition of ASCI initially and additional injury patterns that extended inpatient care.

More recently, a study by Cage et al. described complications associated with high-dose steroid administration in pediatric patients with SCI. ${ }^{2}$ The authors found an overall infection rate of $26 \%$ in the 23 patients treated with highdose steroids and $64 \%$ of the 11 SCI patients not treated with steroids. Respiratory tract infections occurred in $45 \%$ of patients not treated with steroids and in $9 \%$ of patients treated with steroids. Hyperglycemia was present in all patients in both groups. One patient treated with steroids experienced GI bleeding, and wound infection rates were not significantly different. Our pediatric data differed in that $6.8 \%(n=4)$ of the 59 patients treated with steroids and $1.4 \%(n=4)$ of the 295 patients not treated with steroids experienced a respiratory tract infection. The reason for the difference is unclear, but it may be reflective of a short length of stay in our pediatric patient population. The rate of hyperglycemia in our study was $18.6 \%(n=11)$ for 
TABLE 4. Complications by $\mathrm{ASCl}$ and exposure to steroids

\begin{tabular}{|c|c|c|c|c|c|c|c|}
\hline Complication & $\begin{array}{l}\text { ASCI \& Steroids } \\
\quad(n=20)\end{array}$ & $\begin{array}{l}\text { No ASCI \& Steroids } \\
\qquad(n=10)\end{array}$ & $\begin{array}{l}\text { Steroids } \\
(n=30)\end{array}$ & $\begin{array}{l}\text { ASCI \& No Steroids } \\
\quad(n=8)\end{array}$ & $\begin{array}{l}\text { No ASCI \& No Steroids } \\
\qquad(n=50)\end{array}$ & $\begin{array}{l}\text { No Steroids } \\
\quad(n=58)\end{array}$ & $\begin{array}{c}\text { Total } \\
(n=88)\end{array}$ \\
\hline Nausea/vomiting & 13 & 4 & 17 & 3 & 25 & 28 & 45 \\
\hline Hyperglycemia & 6 & 5 & 11 & 1 & 5 & 6 & 17 \\
\hline Bradycardia & 6 & 2 & 8 & 0 & 7 & 7 & 15 \\
\hline Fever & 5 & 2 & 7 & 3 & 19 & 22 & 29 \\
\hline Hypertension & 2 & 3 & 5 & 3 & 13 & 16 & 21 \\
\hline Increased WBC & 2 & 2 & 4 & 0 & 7 & 7 & 11 \\
\hline Respiratory infection & 2 & 2 & 4 & 2 & 2 & 4 & 8 \\
\hline UTI & 2 & 1 & 3 & 0 & 3 & 3 & 6 \\
\hline Wound infection & 2 & 1 & 3 & 1 & 0 & 1 & 4 \\
\hline Bacteremia & 0 & 1 & 1 & 1 & 1 & 2 & 3 \\
\hline Myopathy & 0 & 1 & 1 & 1 & 1 & 2 & 3 \\
\hline Decreased Hb, Hct & 2 & 0 & 2 & 0 & 0 & 0 & 2 \\
\hline Adrenal suppression & 0 & 0 & 0 & 1 & 1 & 2 & 2 \\
\hline Elevated LFTs & 0 & 0 & 0 & 0 & 2 & 2 & 2 \\
\hline Tachycardia & 0 & 0 & 0 & 1 & 0 & 1 & 1 \\
\hline Retroperitoneal bleed & 0 & 0 & 0 & 1 & 0 & 1 & 1 \\
\hline Decubitus ulcer & 1 & 0 & 1 & 0 & 0 & 0 & 1 \\
\hline Seizure & 1 & 0 & 1 & 0 & 0 & 0 & 1 \\
\hline Ileus & 0 & 1 & 1 & 0 & 0 & 0 & 1 \\
\hline Yeast infection & 0 & 0 & 0 & 0 & 1 & 1 & 1 \\
\hline Numbness & 0 & 0 & 0 & 0 & 1 & 1 & 1 \\
\hline Pancreatitis & 0 & 0 & 0 & 0 & 1 & 1 & 1 \\
\hline Total & 44 & 25 & 69 & 18 & 89 & 107 & 176 \\
\hline Average & 2.2 & 2.5 & 2.3 & 2.3 & 1.8 & 1.8 & 2 \\
\hline
\end{tabular}

$\mathrm{Hb}=$ hemoglobin; $\mathrm{Hct}=$ hematocrit; LFTs = results of liver function tests; $\mathrm{UTI}=$ urinary tract infection; $\mathrm{WBC}=$ white blood cell count.

patients treated with steroids and $2.0 \%(\mathrm{n}=6)$ for patients not treated with steroids.

In 2013, the CNS published a Level 1 recommendation against the administration of MPSS for the treatment of ASCI. ${ }^{5}$ The report concludes that Class I, II, and III evidence exists that high-dose steroids are associated with harmful side effects, including death. As a consequence, most sites have now eliminated routine use of MPSS. For these reasons, and the data presented above, our institution is now one of these sites and no longer considers high-dose MPSS a therapy in suspected ASCI.

The current study can be generalizable to other complex protocols for high-risk, low-frequency critical conditions. Leveraging such resources as computerized physician order entry (CPOE) systems and medication infusion smart pumps to facilitate compliance with these protocols is important. Both the new CPOE system and new medication infusion pumps were implemented after the study period and were not available for use during the study period.

The current study has several limitations. This was a retrospective study that required the investigators to use several sources of information, often for each patient. The paper or scanned charts were at times incomplete. We included as many patients (only 32 [5\%] of the initial 602 charts were excluded for incomplete information) and chart data points as possible to learn as much as we could from our historical use of this therapy and relied on what information was included in the charts. Second, while the data collectors were experienced, the authors were unable to differentiate whether complications were attributable to the high-dose MPSS or other traumatic injuries sustained. Third, we were unable to determine the effects of this therapy from a retrospective study design, and thus we did not include patient outcomes in our results. Fourth, although we know that ordering, dosing, and administration was not ideal, we cannot assess whether unintended consequences may have resulted from or potential complications may have been avoided by stopping the intended therapy early or correcting the dosing. However, given the unclear benefits from this therapy, it is possible that no impact would have been realized from either of these protocol deviations.

While our focus was on complications and difficulties with the medication use process, a limitation of our data collection was the absence of patient outcomes. This could be an opportunity for future research in the pediatric population. However, without obvious indication to begin steroid treatment in children with ASCI, it would likely require multicenter collaboration.

\section{Conclusions}

This study highlights an example of managing a com- 
plex, infrequently used medication regimen in the pediatric patient population. As demonstrated by the current study, high-dose MPSS for ASCI may not be able to be delivered to the patient according to the protocol with a high degree of reliability. The findings presented, including complications of steroid use, further support removal of steroids from current practice in pediatric patients with ASCI.

\section{References}

1. American Association of Neurological Surgeons/Congress of Neurological Surgeons Joint Section on Disorders of the Spine and Peripheral Nerves: Pharmacological therapy after acute cervical spinal cord injury. Neurosurgery 50 (Suppl 3):S63-S72, 2002

2. Cage JM, Knox JB, Wimberly RL, Shaha S, Jo C, Riccio AI: Complications associated with high-dose corticosteroid administration in children with spinal cord injury. J Pediatr Orthop 35:687-692, 2015

3. Chang SY, Sevransky J, Martin GS: Protocols in the management of critical illness. Crit Care 16:306, 2012

4. Hurlbert RJ: Methylprednisolone for acute spinal cord injury: an inappropriate standard of care. J Neurosurg 93 (1 Suppl):1-7, 2000

5. Hurlbert RJ, Hadley MN, Walters BC, Aarabi B, Dhall SS, Gelb DE, et al: Pharmacological therapy for acute spinal cord injury. Neurosurgery 72 (Suppl 2): 93-105, 2013

6. Lee HC, Cho DY, Lee WY, Chuang HC: Pitfalls in the treatment of acute cervical spinal cord injury using high-dose methylprednisolone: a retrospect audit of 111 patients. Surgical Neurosurg 68 Suppl 1:S37-S42, 2007

7. Matsumoto T, Tamaki T, Kawakami M, Yoshida M, Ando M, Yamada H: Early complications of high-dose methylprednisolone sodium succinate treatment in the follow-up of acute cervical spinal cord injury. Spine (Phila Pa 1976) 26:426-430, 2001

8. Nesathurai S: Steroids and spinal cord injury: revisiting the NASCIS 2 and NASCIS 3 trials. J Trauma 45:1088-1093, 1998

9. Pettiford JN, Bikhchandani J, Ostlie DJ, St Peter SD, Sharp RJ, Juang D: A review: the role of high dose methylpred- nisolone in spinal cord trauma in children. Pediatr Surg Int 28:287-294, 2012

10. Sayer FT, Kronvall E, Nilsson OG: Methylprednisolone treatment in acute spinal cord injury: the myth challenged through a structured analysis of published literature. Spine J 6:335-343, 2006

11. Short DJ, El Masry WS, Jones PW: High dose methylprednisolone in the management of acute spinal cord injury - a systematic review from a clinical perspective. Spinal Cord 38:273-286, 2000

12. Suberviola B, Gonzalex-Castro A, Llorca J, Ortiz-Melon F, Minambres E: Early complications of high-dose methylprednisolone in acute spinal cord injury patients. Injury 39:748752,2008

\section{Disclosures}

The authors report no conflict of interest concerning the materials or methods used in this study or the findings specified in this paper.

\section{Author Contributions}

Conception and design: all authors. Acquisition of data: Caruso, Daugherty. Analysis and interpretation of data: all authors. Drafting the article: Caruso, Daugherty, Moody. Critically revising the article: all authors. Reviewed submitted version of manuscript: all authors. Approved the final version of the manuscript on behalf of all authors: Caruso. Statistical analysis: Caruso, Daugherty, Moody. Administrative/technical/material support: Caruso, Daugherty, Moody. Study supervision: Caruso.

\section{Supplemental Information \\ Previous Presentations}

Portions of this work were presented as a platform presentation at the 2nd annual meeting of the Pediatric Trauma Society, November 6-7, 2015, in Scottsdale, Arizona.

\section{Correspondence}

Michelle C. Caruso, Division of Pharmacy, Cincinnati Children's Hospital Medical Center, 3333 Burnet Ave., MLC 15010, Cincinnati, OH 45229. email: michelle.caruso@cchmc.org. 